\title{
Estudio analitico arqueomético de dos collares de cuentas de piedra del museo nacional de etnografía y folklore, mediante técnica de FRX-ED
}

DOI: $10.46932 / \mathrm{sjjdv2n1-027}$

Received in: November 1st, 2020

Accepted in: December 30th, 2020

\author{
Juan Villanueva Criales \\ Doctor en Antropología \\ Museo Nacional de Etnografía y Folklore \\ Calle Ingavi 916, zona Central, La Paz, Bolivia \\ E-mail: juan.villanuevacriales@gmail.com
}

Heidi Ruth Mamani Tola

Licenciada en Química Industrial

Universidad del Valle

Avenida Argentina 2083 esq. Nicaragua, La Paz, Bolivia

E-mail: thotbex@gmail.com

Mishka Huanca Cirpa

Licenciada en Física

Universidad Mayor de San Andrés

Calle Boquerón 1122, zona San Pedro, La Paz, Bolivia

E-mail: mishkita26@gmail.com

Primitivo Alanoca Calcina

Técnico en conservaciones de Bienes Culturales

Museo Nacional de Etnografía y Folklore

Calle Ingavi 916, zona Central, La Paz, Bolivia

E-mail: primoalanoca@yahoo.es

José Alfredo Campos Basagoitia

Técnico Restaurador

Museo Nacional de Etnografía y Folklore

Calle Ingavi 916, zona Central, La Paz, Bolivia

E-mail: alfredo.camps@gmail.com

Mario Blanco Cazas

Ingeniero en Geología

Instituto de Investigaciones Geológicas y del Medio Ambiente

Campus Universitario Cota Cota, Calle 27, La Paz, Bolivia

E-mail:mw_bc@hotmail.com

\section{RESUMEN}

Este trabajo presenta los primeros avances en el estudio de cuentas de collar prehispánicas de la colección del Museo Nacional de Etnografía y Folklore (MUSEF) empleando técnica de Fluorescencia de Rayos X por Energía Dispersiva (FRX-ED), en una colaboración interdisciplinaria entre el MUSEF y el Instituto Boliviano de Ciencia y Tecnología Nuclear (IBTEN). Se presentan los métodos, muestra y resultados del análisis FRX-ED de 48 cuentas de collar, agrupadas en dos collares líticos, que fueron analizadas por esta 
técnica no destructiva a fin de avanzar en el conocimiento del origen y conformación de ambos collares. Los resultados permiten sugerir, aunque no confirmar, que la materia prima de ambos collares es lapislázuli, pero sobre todo revelan mayor heterogeneidad química en uno de los collares que en otro. Este resultado permite abrir nuevas preguntas, en torno a si estos dos agrupamientos de cuentas son en efecto fenómenos prehispánicos o ensamblajes posteriores, hechos en tiempos del coleccionismo. Asimismo, denota la necesidad de involucrarse a futuro en estudios de procedencia y en interacciones investigativas con países vecinos.

Palabras clave: arqueometría, materiales líticos, cuentas de collar, FRX-ED.

\section{INTRODUCCION}

El estudio de los materiales líticos prehispánicos en Bolivia empleando técnicas microscópicas y arqueométricas es relativamente temprano, y se centró en el origen de las piedras en que están realizados los monumentos y esculturas del sitio de Tiwanaku. La primera técnica empleada, en este sentido, fue la petrografía sobre secciones delgadas, a cuyos análisis se refiere Arthur Posnansky (1945). La misma, más refinada y con un marco analítico más específico, se volvió a utilizar ya en el contexto de la arqueología nacionalista, en los años sesenta (Ponce Sanginés 1971; Ponce Sanginés y Mogrovejo 1970).

Sin embargo, el uso de técnicas espectrométricas es desde luego más tardío. En el caso de líticos inmuebles, se ha empleado recientemente la técnica de Fluorescencia de Rayos X por energía dispersiva (FRX-ED), técnica no destructiva, para estudiar las canteras de origen de las andesitas y sobre todo las areniscas de los monumentos de Tiwanaku (Janusek et al 2013). En cuanto a los artefactos líticos portátiles, su estudio se ha beneficiado también de las nuevas técnicas disponibles, especialmente en cuanto se refiere a las puntas de flecha trabajadas en obsidiana. La técnica de espectroscopia por Activación Neutrónica (NAA) ha sido empleada para este cometido tanto en estudios regionales (Burger et al 2000) como en otros vinculados específicamente a los materiales de Tiwanaku (Giesso 2003).

Como se puede observar de este breve recuento de antecedentes, el trabajo arqueométrico sobre ítems líticos se ha centrado en el fenómeno social Tiwanaku, y se ha realizado fundamentalmente como parte de proyectos extranjeros por la ausencia de la mayor parte de estas técnicas en Bolivia. Sin embargo, desde 2014 el Museo Nacional de Etnografía y Folklore se ha equipado con un espectrómetro portátil de FRX-ED, iniciando una importante interacción con el Instituto Boliviano de Ciencia y Tecnología Nuclear (IBTEN) para el tratamiento de datos y el avance de la investigación arqueométrica en el país, uno de cuyos frutos es el presente trabajo. Asimismo, este trabajo es novedoso por considerar la industria de la lapidaria o cuentas de collar, largamente descuidada por los trabajos previos, y por trabajar con especímenes de museo empleando técnicas no destructivas.

Como es el caso en muchos museos del mundo, las colecciones de objetos arqueológicos del MUSEF han sido formadas en gran medida su por donaciones tempranas de coleccionistas privados $u$ 
otros entes no académicos, y por tanto los objetos que las integran carecen de contextos arqueológicos certeros. Esto implica que no es posible asumir que agrupamientos de ítems similares como cuentas de collar hayan sido formados en tiempos prehispánicos, pudiendo haber sido recolectados y reunidos por coleccionistas posteriormente. Para resolver estas preguntas, es imprescindible el conocimiento profundo de los materiales que componen las cuentas de collar, y este trabajo es un primer paso, aún preliminar, en ese sentido.

En lo que sigue, se presentan la muestra, métodos y resultados de una investigación mediante técnica de FRX-ED para la caracterización arqueométrica de 48 cuentas de collar de piedra, en dos especímenes de la colección de materiales arqueológicos inorgánicos del MUSEF.

\section{MÉTODO Y MUESTRA}

Actualmente en el mundo ha surgido la utilización de la física nuclear con fines pacíficos, específicamente en el campo de las radiaciones ionizantes. Entre las muchas utilidades de estas técnicas se encuentran los estudios analíticos sobre objetos del patrimonio cultural y de contextos arqueológicos. Estas técnicas son utilizadas para determinar la composición elemental de los materiales, permitiendo detectar similitudes y diferencias al interior de conjuntos de piezas arqueológicas, y/o entre piezas y determinadas fuentes de materiales. Estos resultados pueden permitir, a la larga, tratar temáticas de movilidad, influencia e intercambio.

La fluorescencia de rayos X de energía dispersiva (FRX-ED) es una técnica analítica no destructiva que permite la identificación de elementos químicos presentes en objetos patrimoniales, sin preparación previa de la muestra, lo que la convierte en una excelente técnica para analizar objetos de interés histórico y cultural.

En términos técnicos, la fluorescencia de rayos $\mathrm{X}$ es un método de caracterización basado en las interacciones de las radiaciones con la materia (Moens et al. 2000; Garrison 2003). Se basa en la emisión por efecto fotoeléctrico de rayos $\mathrm{X}$ característicos de los elementos que constituyen un material cuando éste es irradiado con un haz de rayos X. La espectroscopia de fluorescencia excita la muestra mediante la absorción de fotón de luz desde su estado electrónico basal a uno de los distintos estados vibracionales del estado electrónico excitado. Las colisiones con otras moléculas causan que la molécula excitada pierda su energía hasta que alcanza el estado vibracional más bajo del estado electrónico excitado. La molécula desciende luego a uno de los distintos niveles de vibración del estado electrónico basal, emitiendo un fotón en el proceso. Como las moléculas pueden caer a cualquiera de los diferentes niveles de vibración en el estado basal, los fotones emitidos tendrán diferente energía y por lo tanto frecuencias. 
Así pues, mediante el análisis de las diferentes frecuencias de luz emitida por espectrometría de fluorescencia, junto con sus intensidades relativas se puede determinar la estructura de los diferentes niveles de vibración.

Los rayos $\mathrm{X}$ poseen una energía tal que al incidir sobre la muestra puede ionizarla expulsando electrones de la capa más interna. Estos iones son altamente inestables y esas vacantes son ocupadas por electrones de capas superiores. Estos saltos de electrones de un nivel de mayor energía a uno de menor, desprenden una radiación característica que es la fluorescencia de rayos X. El análisis cualitativo se obtiene a partir de la energía característica de la radiación de FRX emitida, el análisis cuantitativo se obtiene por conteo de los rayos $\mathrm{X}$.

En este trabajo, se empleó un espectrómetro portátil Niton XL3t Gold. El tubo de rayos X genera el haz primario que irradia la muestra y excita la radiación de fluorescencia de modo continuo, cubriendo un amplio intervalo espectral con altos voltajes e intensidades de corriente controlables con pequeños incrementos en el rango de operación que oscila entre $1-50 \mathrm{kV}$ y entre $10-200 \mu \mathrm{A}$, lo cual permite excitar todos los elementos presentes en la muestra. Se utilizó la calibración predeterminada Test All Geo, con disparos de 240 segundos separados en cuatro series de 60 .

Para el tratamiento de datos de los espectros con los softwares NDT y QXAS, se hizo una comparación de los resultados de FRX-ED en relación con aquellos obtenidos mediante la lectura de los estándares conocidos de granito, grnodiorita y basalto. Los elementos con mayor coeficiente de determinación (>0.9) son los que se muestra en el cuadro de estandarización del método (Tabla 1), los cuales fueron considerados para este trabajo.

Tabla 1. Estandarización del método NDT/QXAS

\begin{tabular}{|c|c|c|c|c|c|c|}
\hline \multirow{3}{*}{$\frac{\text { Elemento }}{\mathrm{Mg}}$} & \multicolumn{3}{|c|}{ NDT } & \multicolumn{3}{|c|}{ Qxas } \\
\hline & \multicolumn{2}{|c|}{ Pendiente } & \multirow{2}{*}{$\begin{array}{c}\text { Coef. Determinación } \\
0.98\end{array}$} & \multicolumn{2}{|c|}{ Pendiente } & \multirow{2}{*}{$\begin{array}{c}\text { Coef. Determinación } \\
0.97\end{array}$} \\
\hline & $1.83 \pm$ & 0.05 & & 1.32 & \pm 0.07 & \\
\hline $\mathrm{Al}$ & $1.56 \pm$ & 0.07 & 0.88 & 1.03 & \pm 0.04 & 0.97 \\
\hline $\mathrm{Si}$ & $1.39 \pm$ & 0.05 & 0.87 & 0.89 & \pm 0.08 & 0.76 \\
\hline $\mathrm{P}$ & $4.15 \pm$ & 0.43 & 0.79 & 2.5 & \pm 0.59 & 0.69 \\
\hline $\mathrm{S}$ & $1.81 \pm$ & 0.07 & 0.98 & 1.01 & \pm 0.05 & 0.98 \\
\hline K & $0.93 \pm$ & 0.01 & 0.99 & 1.01 & \pm 0.03 & 0.99 \\
\hline $\mathrm{Ca}$ & $1.24 \pm$ & 0.01 & 0.99 & 0.92 & \pm 0.04 & 0.99 \\
\hline $\mathrm{Ti}$ & $1.04 \pm$ & 0.03 & 0.97 & 1.22 & \pm 0.08 & 0.95 \\
\hline V & $1.2 \pm$ & 0.07 & 0.83 & 1.11 & \pm 0.09 & 0.93 \\
\hline $\mathrm{Cr}$ & $0.86 \pm$ & 0.01 & 0.99 & 0.73 & \pm 0.03 & 0.85 \\
\hline $\mathrm{Mn}$ & $0.79 \pm$ & 0.03 & 0.95 & 1.14 & \pm 0.06 & 0.98 \\
\hline $\mathrm{Fe}$ & $1.06 \pm$ & 0.01 & 0.99 & 1 & \pm 0.15 & 0.79 \\
\hline $\mathrm{Ni}$ & 0.7 & 0.01 & 0.99 & & & \\
\hline $\mathrm{Cu}$ & $0.99 \pm$ & 0.1 & 0.63 & & & \\
\hline
\end{tabular}




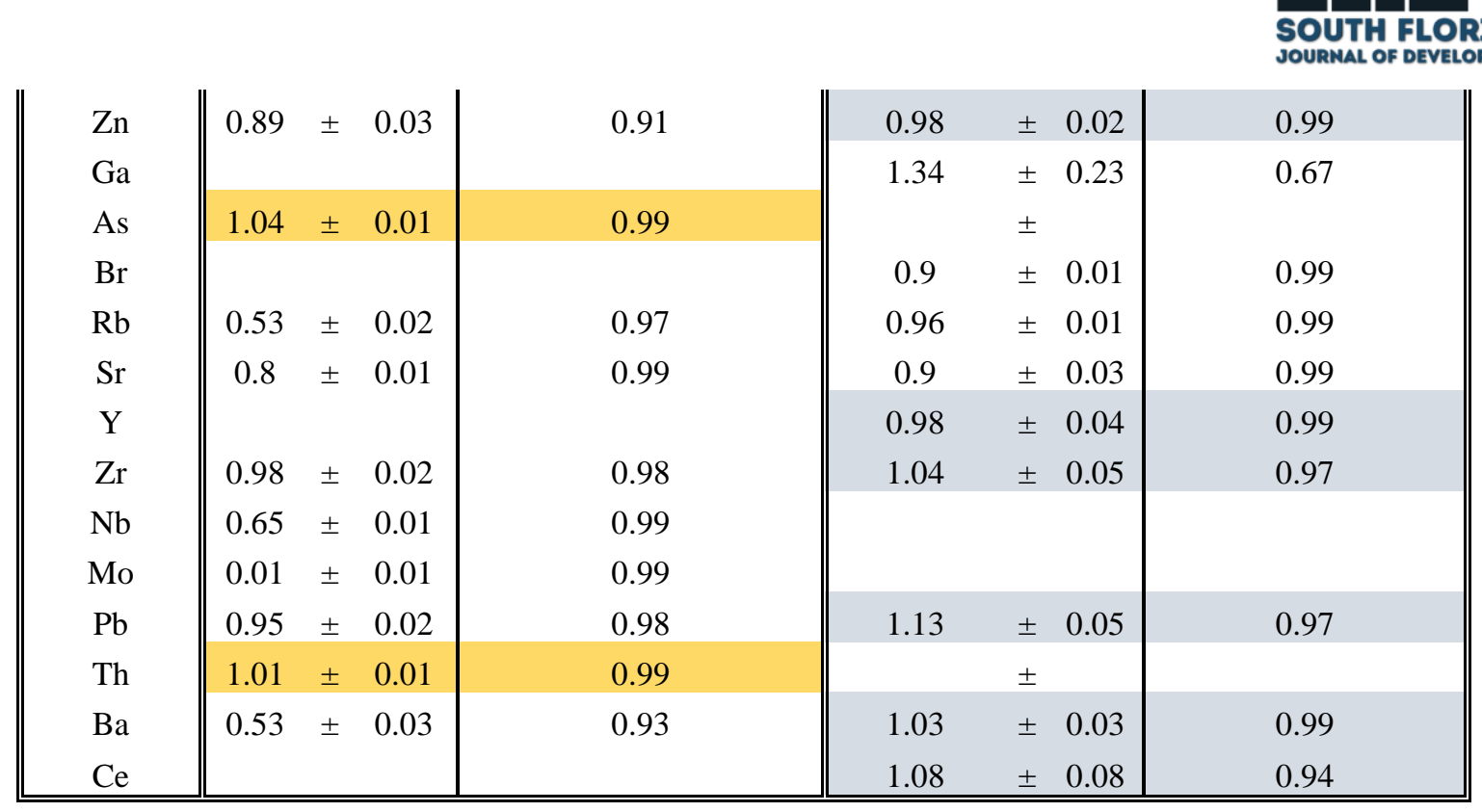

*Coef. Determinación $>90 \%$

$* 1.02 \leq$ Pendiente $\leq 1.62$

Posteriormente, los resultados fueron expresados en gráficos de barras y también se acudió al análisis de conglomerados para intentar detectar agrupamientos y diferencias al interior de las muestras.

La muestra estudiada consistió en 48 cuentas de collar de piedra azul con jaspes blancos, organizadas en dos collares (ID 20381 e ID 10097) (Figura 1). Lamentablemente, ambas piezas carecen de contexto: el collar 20381, que denominaremos Collar 1, fue donado por un coleccionista privado el año 2004, y consta exclusivamente de cuentas tubulares más una cuenta central triangular, con perforación en la parte superior. El collar 10097, que denominaremos Collar 2, fue adquirido en los años 90, aunque se desconoce mayor información al respecto. Consta mayormente de cuentas tubulares, intercaladas con algunas circulares. Se analizaron 29 cuentas del Collar 1, y 19 cuentas del Collar 2.

Figura 1. El Collar 1 o ID 20381 (izq.) y el Collar 2 o ID 10097 (der.)
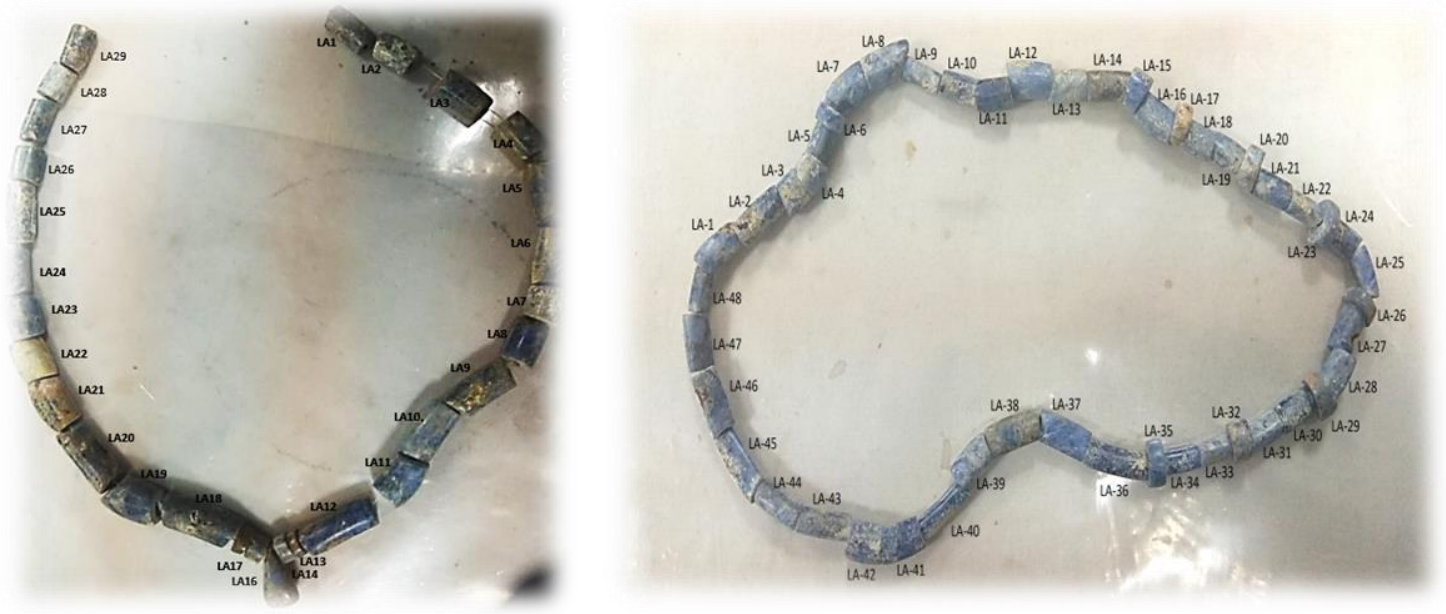


\section{RESULTADOS}

Una apreciación general sobre el total de la muestra (Tablas 2 y 3), es la presencia constante de altas proporciones de Aluminio $(\mathrm{Al})$ y Silicio $(\mathrm{Si})$, que de hecho fueron omitidos de los gráficos porque impedían visualizar adecuadamente el aporte del resto de los elementos. Los otros elementos que aparecen constantemente en la muestra son el Calcio (Ca), Azufre (S) y Hierro (Fe).

Tabla 2. Resultados del análisis del Collar 1

\begin{tabular}{|c|c|c|c|c|c|c|c|c|c|c|c|}
\hline Muestras & $\mathbf{S r}$ & $\mathbf{Z n}$ & $\mathrm{Cu}$ & $\mathbf{N i}$ & $\mathbf{F e}$ & Mn & $\mathrm{Cr}$ & Ca & $\mathbf{S}$ & Cl & Mg \\
\hline LA-1 & 298.2 & 383.9 & 217.8 & 118.2 & 13312.4 & 360.6 & 72.2 & 17968.0 & 5556.3 & 11606.7 & 11680.9 \\
\hline LA-2 & 695.0 & 1546.1 & 478.8 & 205.9 & 51947.3 & 1336.7 & 340.1 & 32125.0 & 5337.1 & 5989.9 & 12804.5 \\
\hline LA-3 & 57.1 & 189.2 & 285.5 & 121.8 & 24881.0 & 1366.5 & 84.0 & 19799.7 & 6244.1 & 6144.6 & 12066.9 \\
\hline LA-4 & 3.3 & 40.5 & 250.9 & 94.6 & 1153.4 & & 69.6 & 20317.1 & 4874.8 & 13232.1 & 10906.5 \\
\hline LA-5 & 68.4 & 29.7 & 209.6 & 133.7 & 1090.2 & & 81.0 & 5730.8 & 5050.9 & 17597.0 & 7737.6 \\
\hline LA-6 & 171.6 & 2.2 & 217.8 & 103.5 & 4946.0 & 164.6 & 85.6 & 10867.2 & 5685.3 & 5389.8 & 10056.4 \\
\hline LA-7 & 33.7 & 514.1 & 253.1 & 69.1 & 1782.2 & & 65.1 & 14437.9 & 6335.5 & 20918.5 & 10136.1 \\
\hline LA-8 & 411.7 & 880.7 & 107.1 & 99.3 & 14489.3 & 5451.0 & 81.8 & 20846.7 & 5783.5 & 22330.0 & 10249.2 \\
\hline LA-9 & & & & 87.1 & & & 55.1 & & & 14787.6 & 78.2 \\
\hline LA-10 & 42.5 & 98.8 & 106.5 & 104.3 & 3061.6 & 168.4 & 30.8 & 9869.3 & 4185.6 & 42385.6 & 13268.2 \\
\hline LA-11 & 561.5 & 553.6 & 97.6 & 95.6 & 3976.6 & & 49.1 & 13438.9 & 3978.9 & 13385.0 & 7993.7 \\
\hline LA-12 & & 82.9 & 112.2 & 114.7 & 2026.2 & & 67.1 & 10110.2 & 4751.0 & 2398.1 & 11505.5 \\
\hline LA-15 & 1.1 & 115.7 & 205.4 & 108.6 & 1482.2 & & 42.6 & 11709.7 & 4324.1 & 36962.5 & 14104.8 \\
\hline & & & & 269.1 & 88600.6 & & 224.5 & & & 3.2 & 19866.4 \\
\hline LA-19 & 129.2 & 185.3 & 100.8 & 96.9 & 785.7 & & 55.7 & 11768.1 & 5019.5 & 12036.3 & 8733.2 \\
\hline LA-20 & 122.0 & 685.8 & 147.4 & 102.4 & 4584.4 & & 50.9 & 12730.4 & 3800.5 & 9088.8 & \\
\hline LA-21 & 162.9 & 376.2 & & 102.7 & 5107.7 & 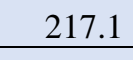 & 49.8 & 13634.4 & 4016.9 & 8338.8 & 7775.1 \\
\hline LA-22 & 28.5 & & 123.2 & 87.1 & 2682.8 & & 65.2 & 6124.0 & 5567.4 & 7139.1 & 10025.2 \\
\hline LA-23 & & 126.7 & & 4326.7 & 2.5 & & 9.6 & 129 & .4 & 8.8 & 65618.4 \\
\hline & & 845.8 & 00.0 & 133.1 & 12500.0 & & 60.5 & 10030.3 & 4000.6 & 20026.3 & 9919.2 \\
\hline LA-25 & 127.8 & 1051.1 & 131.5 & 101.3 & 5415.9 & 1370.7 & 70.9 & 8648.2 & 4588.2 & 13936.4 & 10228.7 \\
\hline LA-26 & 20.7 & & 181.7 & 148.4 & 1481.8 & & 56.6 & 14040.8 & 4983.1 & 11010.2 & 11048.3 \\
\hline LA-27 & 157.4 & 1605.6 & 277.3 & 96.2 & 3220.7 & & 65.0 & 9084.8 & 6469.6 & 11388.2 & 10652.0 \\
\hline LA-28 & 224.3 & & 78.5 & 99.7 & 4074.6 & & 113.0 & 13769.3 & 5779.1 & 14900.5 & 13318.1 \\
\hline LA-29 & 154.4 & & 237.0 & & & & 96.1 & 12073.8 & 5629.2 & 2859.1 & 11290.3 \\
\hline
\end{tabular}

Tabla 3. Resultados del análisis del Collar 2

\begin{tabular}{|c|c|c|c|c|c|c|c|c|c|c|c|}
\hline Muestras & $\mathrm{Sr}$ & Zn & $\mathrm{Cu}$ & $\mathrm{Ni}$ & $\mathrm{Fe}$ & Mn & $\mathrm{Cr}$ & Ca & $\mathbf{S}$ & Cl & Mg \\
\hline LA-1 & 21.3 & 223.0 & 74.4 & & & & 57.6 & 2180.1 & 9937.7 & 10899.2 & 5881.6 \\
\hline LA-2 & 89.4 & & 50.7 & & 9711.6 & 364.6 & 68.9 & 2662.1 & 8554.8 & 24120.8 & 7729.9 \\
\hline LA-11 & 23.0 & 1196.8 & 71.1 & & 3682.2 & & 52.2 & 3549.7 & 8798.8 & 44424.9 & 9772.3 \\
\hline LA-14 & 165.9 & 148.7 & 67.2 & & 1192.7 & & 71.3 & 27839.6 & 8013.8 & 8909.8 & 10903.8 \\
\hline LA -29 & & & 4346.5 & & & & & & 57205.9 & 22269.2 & 91455.5 \\
\hline LA-36 & 72.8 & 153.7 & 68.8 & & 11555.9 & & 127.0 & 1932.4 & 11690.0 & 12272.6 & 5929.3 \\
\hline LA-40 & 38.7 & & 56.2 & 93.9 & 345.7 & & 37.7 & 2013.5 & 9742.0 & 18081.3 & 5305.9 \\
\hline
\end{tabular}




\begin{tabular}{|l|r|r|r|r|r|r|r|r|r|r|r|} 
LA-3 & 285.5 & 321.0 & 251.1 & 86.4 & & & 68.3 & 7623.1 & 4921.2 & & 10912.6 \\
\hline LA-8 & 57.5 & & 48.2 & & & & 70.1 & 5660.6 & 4715.3 & 40829.3 & 9711.8 \\
\hline LA-15 & 329.0 & & 65.5 & 134.3 & & & 34.3 & 5643.3 & 5874.5 & 34351.7 & 7995.4 \\
\hline LA 4 & 39.4 & 136.9 & 55.4 & & & & 65.3 & 12832.3 & 7358.1 & 20859.2 & 9888.4 \\
\hline LA 5 & 51.8 & 197.3 & 56.7 & & 403.7 & & 52.1 & 6991.2 & 6050.4 & 34848.7 & 10229.8 \\
\hline LA 7 & 91.7 & 115.1 & 49.9 & & 1296.1 & & 93.5 & 8452.1 & 5027.6 & 39110.4 & \\
\hline LA 8 & 75.3 & & 48.4 & 80.5 & & & 63.4 & 4454.1 & 6843.1 & 22817.9 & \\
\hline LA-35 & 90.5 & & 113.8 & 160.8 & 2310.5 & & & 5825.6 & 5513.4 & 36250.0 & \\
\hline LA-37 & 61.0 & & 52.9 & & 2224.4 & & 85.2 & 2861.3 & 5367.1 & 62418.9 & \\
\hline LA-13 & 98.0 & & 43.8 & & 685.6 & & 62.4 & 8935.5 & 6192.8 & 15954.0 & \\
\hline LA-30 & 157.5 & 714.3 & 43.4 & 138.3 & 2578.4 & & 26.4 & 2433.4 & 3489.8 & 60010.1 & \\
\hline LA-41 & 110.0 & 226.3 & 40.3 & & 1948.8 & & 60.8 & 3300.3 & 9021.1 & 20298.7 & \\
\hline
\end{tabular}

Es importante notar que la presencia de estos cinco elementos (Al, Si, CA, S, Fe) permite sugerir que la piedra azul en cuestión se trata de lapislázuli. El lapislázuli es una roca compuesta por tres minerales: Lazurita $\left(\mathrm{Na}_{3} \mathrm{Ca}\left(\mathrm{Si}_{3} \mathrm{Al}_{3}\right) \mathrm{O}_{12} \mathrm{~S}\right)$, Calcita $\left(\mathrm{CaCO}_{3}\right)$ y Pirita $\left(\mathrm{FeS}_{2}\right)$, que le otorgan sus jaspes azul, blanco y dorado, respectivamente. Sin embargo, cabe aclarar que los resultados al respecto no son concluyentes, y que un estudio con otras técnicas como petrografía o difracción de rayos x (ambas forzosamente destructivas al menos desde la disponibilidad técnica del medio boliviano), permitirían la caracterización mineralógica de la lazurita y los demás minerales de modo más confiable.

El Cloro $(\mathrm{Cl})$ y Magnesio $(\mathrm{Mg})$ son otros elementos comunes a toda la muestra, aunque el cloro podría presentarse debido a interacción con el suelo del entorno, al tratarse de un elemento hidrosoluble. Finalmente, se ha podido detectar confiablemente Estroncio ( $\mathrm{Sr}$ ) en proporciones muy variables, y Manganeso (Mn), Cinc (Zn) y Níquel (Ni) en ciertos ejemplares concretos.

Ingresando al análisis del primer collar, se puede evidenciar por la gráfica (Figura 2) que las muestras son bastante heterogéneas, siendo las proporciones de $\mathrm{Fe}, \mathrm{Mg}$ y $\mathrm{Mn}$ algunos de los aspectos que mayor variabilidad provocan.

Figura 2. Resultados del análisis elemental sobre cuentas del Collar 1

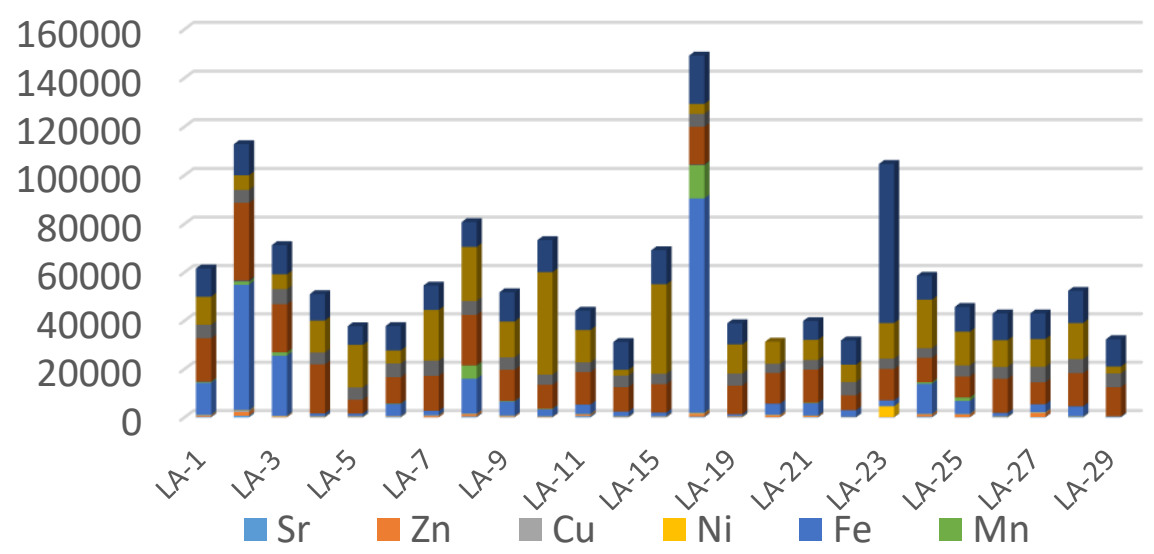


Se ha efectuado gráficos de correlación de las variables resultantes de un análisis de componentes principales (ACP), que permite notar cuatro grupos bastante discretos, además de un par de "otros" que difieren mucho del común de las cuentas analizadas (Figura 3).

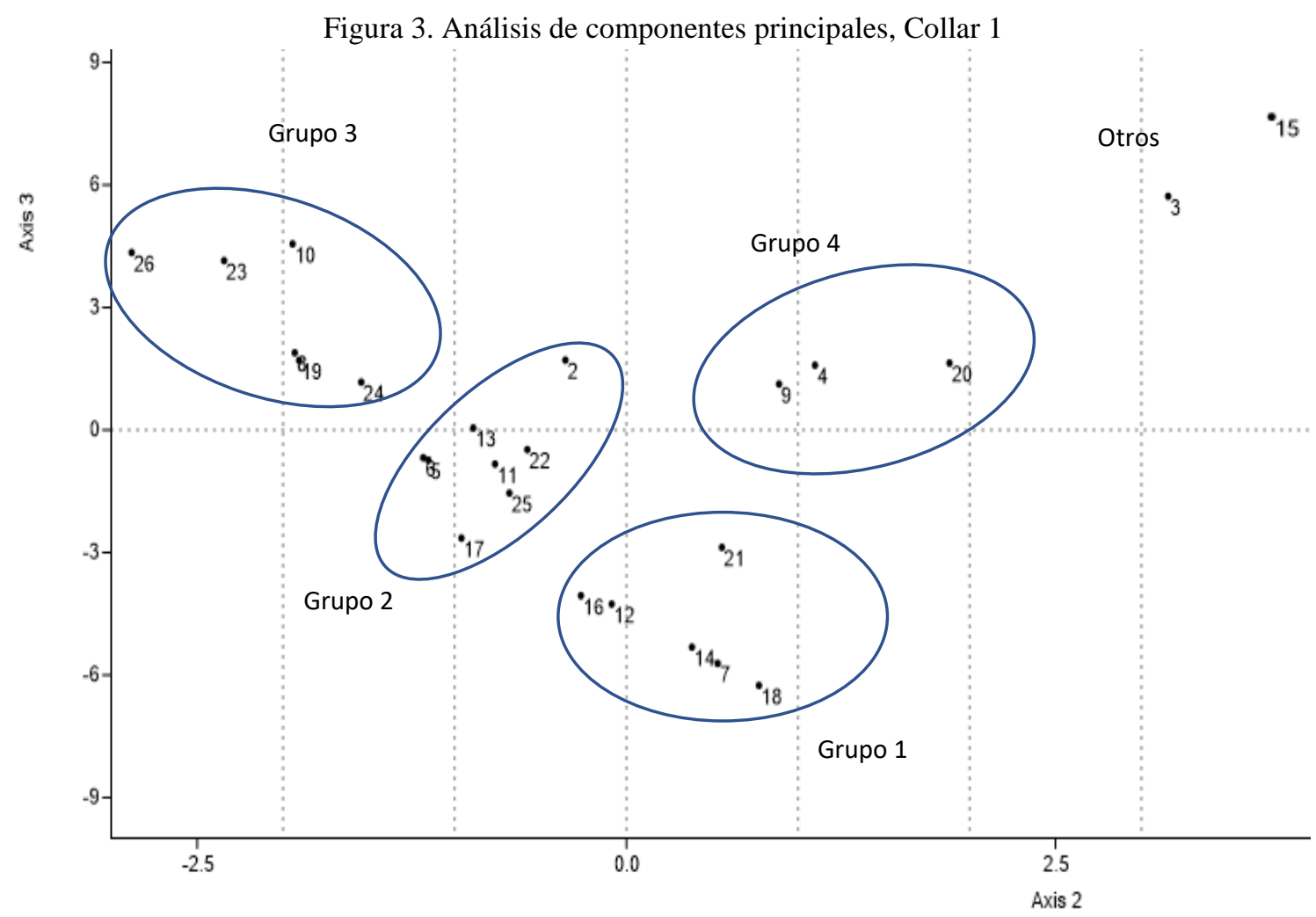

Revisando a detalle los valores de las cuentas agrupadas según el análisis de componentes, se puede ver que tienden a distinguirse por proporciones variables, especialmente de Fe y Mg (Figura 4). Sin embargo, al examinar las cuentas de collar en detalle, no presentan diferencias macroscópicas notables respecto del resto de la muestra, ni en color del material ni en dimensiones o técnica de elaboración.

En cuanto al segundo collar, a diferencia del primero sus cuentas incorporaban variedad significativa de tonos de color azul. Se decidió agrupar previamente las cuentas según su color en tres tonalidades: azul oscuro, celeste y celeste claro (Figura 5). 
Figura 4. Resultados por grupo, Collar 1

Primer Grupo

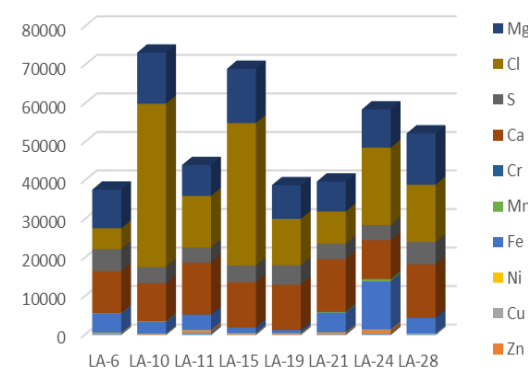

Tercer Grupo

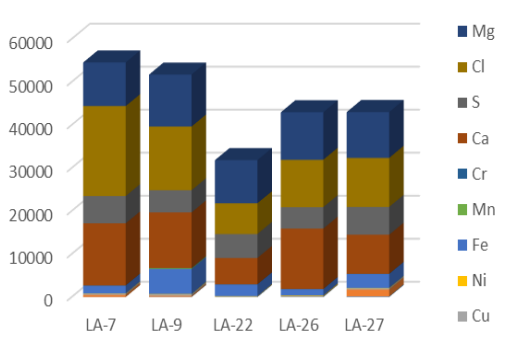

Segundo Grupo
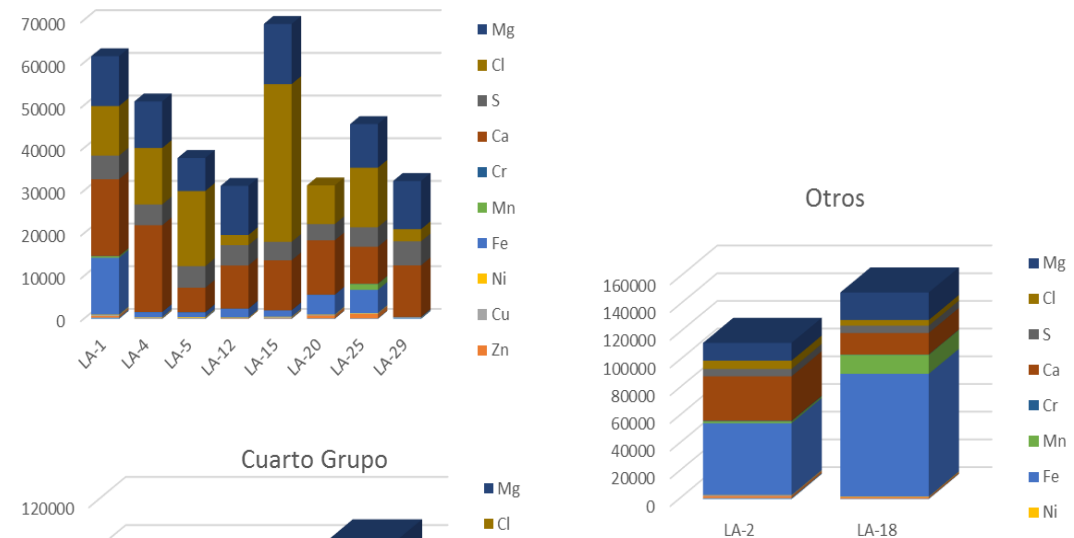

Figura 5. Resultados del análisis de cuentas del Collar 2 agrupadas por color
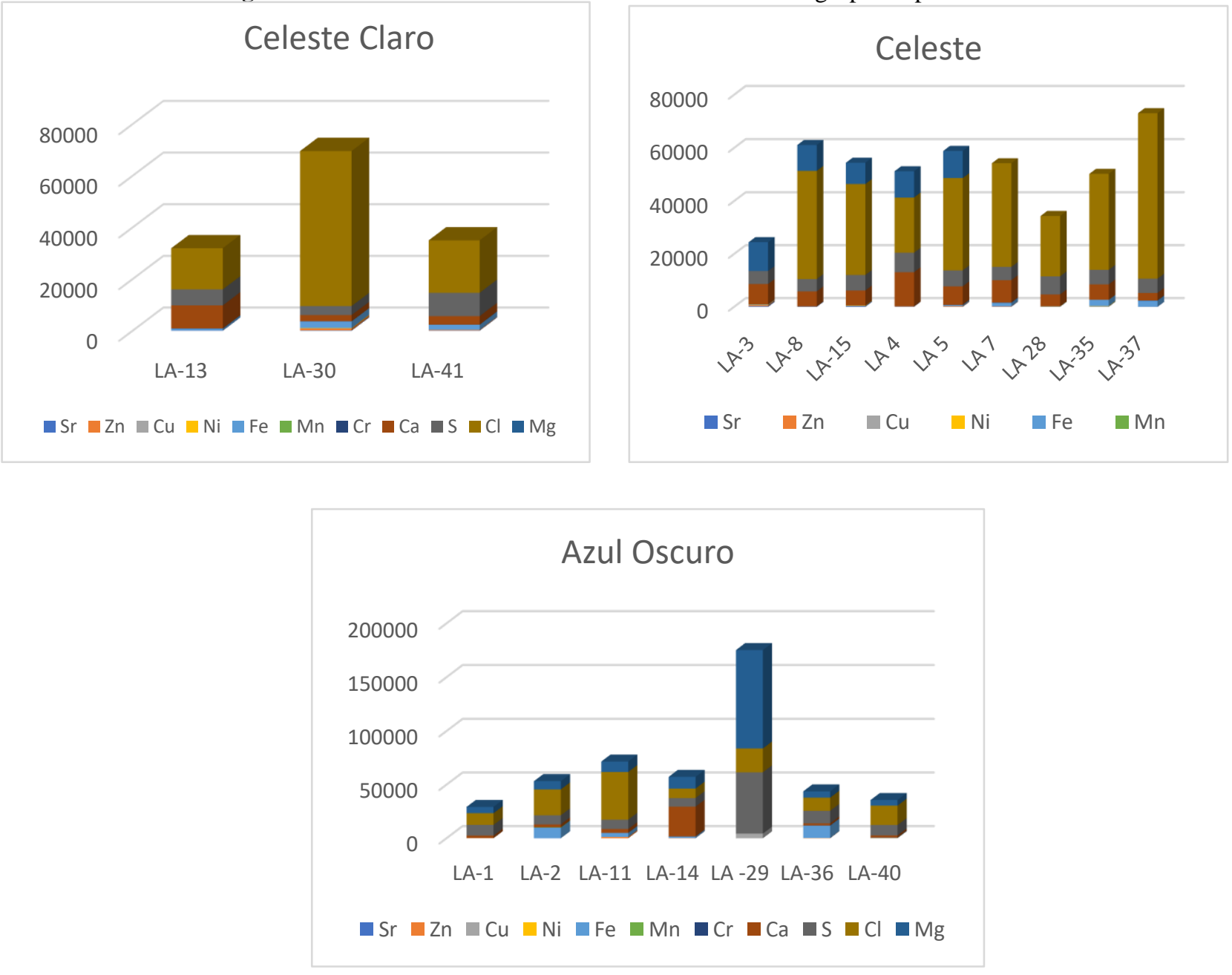
$\mathrm{Al}$ revisar los resultados del análisis agrupado según colores, resulta bastante claro que la mayor o menor presencia de Mg incide en la intensidad de la coloración azul de las cuentas de collar. Sin embargo, a la hora de realizar el análisis de conglomerados la muestra da la impresión de ser químicamente más homogénea, con las lecturas tendiendo a formar una nube uniforme donde es difícil identificar agrupamientos claros (Figura 6).

Figura 6. Análisis de componentes principales, Collar 2

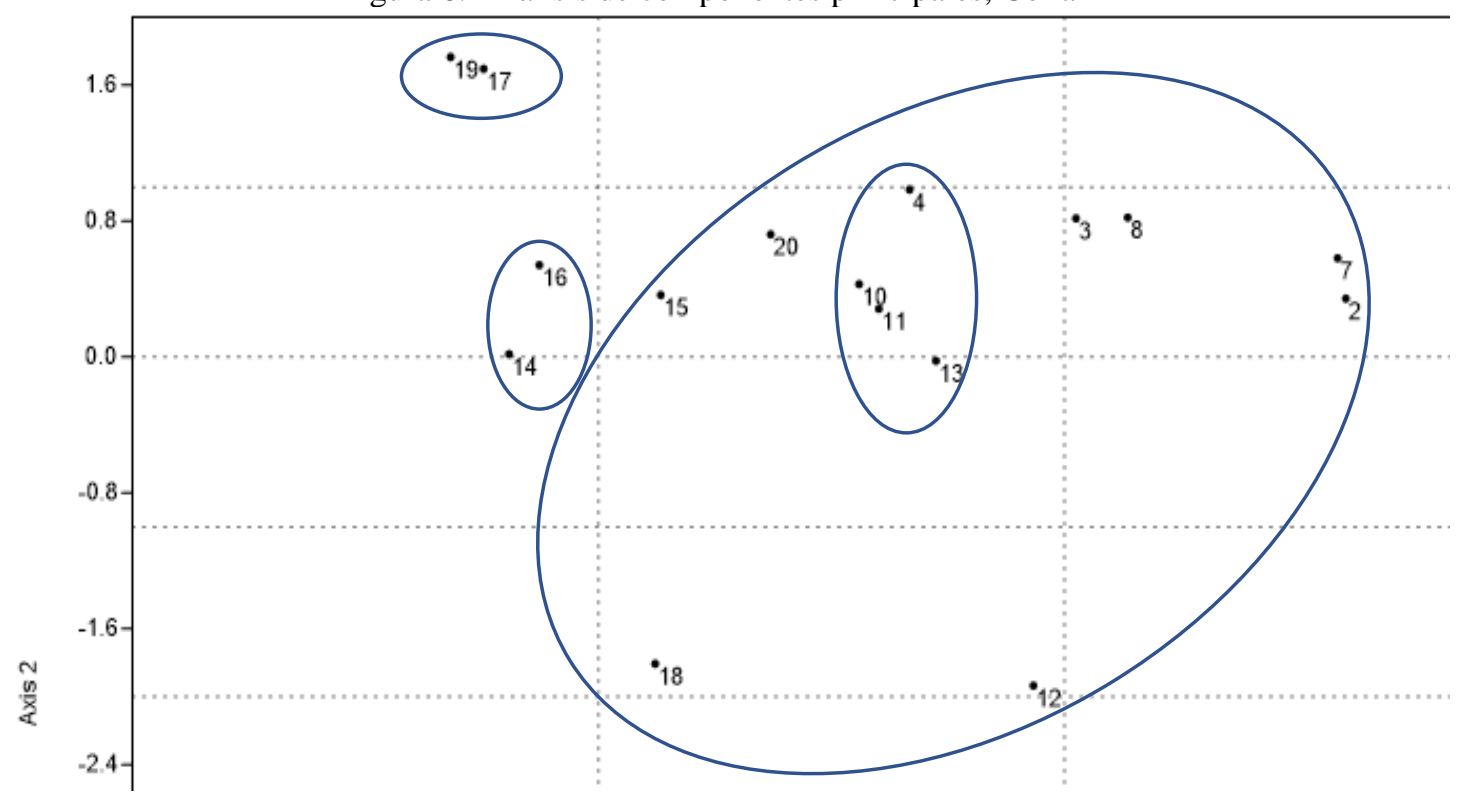

\section{CONCLUSIONES}

El análisis elemental realizado con FRX-ED permitió sugerir, a partir de los datos elementales obtenidos, al lapislázuli como el material en que se habrán elaborado las cuentas de ambos collares. Desde luego, solo una caracterización mineralógica por otras técnicas arqueométricas podrá corroborar definitivamente esta suposición.

El estudio del Collar 2 permite sugerir que la proporción de magnesio influye en las tonalidades de azul del material, apoyando la idea de que el color de estas cuentas se debe a la materia prima y no a procesos tafonómicos, pátinas orgánicas o procedimientos de conservación.

En el estudio del Collar 1 se advirtió mucha más variedad elemental entre cuentas que en el Collar 2. En efecto, en el primer caso se dibujan unos agrupamientos mucho más discretos y claros que en el segundo.

Este fenómeno implica pensar, por un lado, en el origen de esta variabilidad química, que podría deberse a variabilidad interna a una sola cantera de materia prima o a diversas canteras espacialmente distantes. Comenzar a atacar estas preguntas a futuro implicará interactuar con los avances arqueométricos 
sobre la materia en países vecinos, fundamentalmente en Chile, en cuya región norte se conoce la existencia de lapislázuli y materiales similares (Westfall et al 2007).

Por otro lado, la diferencia entre ambos collares plantea diversos escenarios posibles. El primero, que la misma se deba a fenómenos prehispánicos, es decir que un collar fue realizado con materiales de una sola cantera o de una cantera más homogénea, mientras que el otro fue realizado con lapislázuli de diversas canteras o de una cantera más heterogénea. El segundo escenario, que no puede descartarse debido a la falta de contexto de las piezas, es que el collar más heterogéneo sea una colección moderna de cuentas de lapislázuli de diversas canteras, y el otro un collar completo de origen prehispánico. Los parecidos en tamaño y técnica de manufactura de las cuentas de ambos collares en cuestión permiten dudas sobre esta última hipótesis, pero es ciertamente llamativo que un collar sea tanto más heterogéneo que el otro. Claramente, no es posible asumir que ambos colares son agrupamientos de origen prehispánico.

De una u otra manera, es imprescindible ampliar los estudios arqueométricos de lapidaria arqueológica y estrechar lazos transfronterizos en la procura de aclarar fenómenos de interacción prehispánica. Asimismo, será de mucho interés incursionar en técnicas espectrométricas de caracterización mineral. 


\section{REFERENCIAS}

BURGER, Richard L., Karen L. MOHR CHÁVEZ y Sergio J. CHÁVEZ. 2000. Through the Glass Darkly: Prehispanic Obsidian Procurement and Exchange in Southern Peru and Northern Bolivia. Journal of World Prehistory 14(3), 267-362.

CONTRERAS, Ricardo, Ramón IXQUIAC y Osmar HERNÁNDEZ. 1999. Análisis multielemental por el método de fluorescencia de rayos x en muestras arqueológicas y geológicas de, Guatemala. Proyecto No. 48-98, Departamento de Física, Universidad de San Carlos de Guatemala.

FERNÁNDEZ, Ramón. 1995. Análisis de cerámicas arqueológicas mediante fluorescencia de rayos x por reflexión total y contrastación mediante análisis por activación de neutrones. Ponencia presentada al I Congreso Nacional de Arqueometría y Arqueología, Universidad Autónoma de Madrid, enero 1995. DOI: 10.13140/RG.2.1.2259.2485

GIESSO, Martín. 2003. Stone Tool Production in the Tiwanaku Hertland. En Tiwanaku and its Hinterland 2: Urban and Rural Archaeology (editado por Alan Kolata), pp. 363-383). Smithsonian Institution Press, Washington y Londres.

JANUSEK, John Wayne, Patrick Ryan WILLIAMS, Mark GOLITKO y Carlos LÉMUZ AGUIRRE. 2013. Building Taypikala: Telluric Transformations in the Lithic Production of Tiwanaku. En Mining and Quarrying in the Ancient Andes. Sociopolitical, Economic, and Symbolic Dimensions (editado por Nicolás Tripcevich y Kevin J. Vaughn), pp. 65-98. Springer, Nueva York.

PONCE SANGINÉS, Carlos. 1971. Examen arqueológico de las ruinas Precolombinas de Tiwanaku. En Procedencia de las areniscas utilizadas en el temple Precolombino de Pumapunku (Tiwanaku), 22, pp. 13-206. Academia Nacional de Ciencias de Bolivia, La Paz.

PONCE SANGINÉS, Carlos y Gerardo MOGROVEJO TERRAZAS. 1970. Acerca de la Procedencia del material lítico de los monumentos de Tiwanaku. Academia Nacional de Ciencias de Bolivia, La Paz. POSNANSKY, Arthur. 1945. Tihuanacu. La Cuan del Hombre Americano (Tomo II). J.J: Augustin, Nueva York.

WESTFALL, Catherine, Mauricio BELMAR y Carlos GONZÁLEZ. 2007. Aplicación geo-arqueológica de microscopía electrónica y microsonda en piezas metalográficas y líticas del cementerio Regimiento Chorrillos de Calama. Actas del XVII Congreso Nacional de Arqueología Chilena, Valdivia 2006 (N. 2), 835-846. 